MATEC Web of Conferences 22,01043 (2015)

DOI: $10.1051 /$ matec conf/ 20152201043

(C) Owned by the authors, published by EDP Sciences, 2015

\title{
Signal Interference between GPS and Wide Area Augmentation System
}

\author{
Wei Liu* \\ Merchant Marine College, Shanghai Maritime University, Shanghai, China
}

\begin{abstract}
With the development of Global Navigation Satellite System (GNSS), multiple constellations broadcasting more signals in the same frequency bands will cause interference effects on the systems. At a minimum, all GNSS signals and services must be compatible. In this paper, a complete methodology for the signal interference assessment is presented. Real simulations accounting for the interference effects were carried out on the L1 band where GPS and Wide Area Augmentation System (WAAS) are sharing the same band. And the results show that the WASS can provide a sound basis for compatibility with GPS.
\end{abstract}

Keywords: satellite navigation; signal interference; simulation model

\section{INTRODUCTION}

An increasing number of new Global Navigation Satellite System (GNSS) signals lead to the need to assess the radio frequency compatibility carefully. And the GNSS radio frequency compatibility has become a matter of great concern for the system providers and user community. Compatibility refers to the ability of global and regional navigation satellite systems and augmentations to be used separately or together without causing unacceptable interference and/or other harms to an individual system and/or service [1]. In order to make open signals and services interoperable and maximize benefit to all GNSS users, all GNSS signals and services must be compatible at first.

Three different approaches (which are field testing, computer simulations and analytical techniques) have been developed in order to assess the intrasystem interference (interference from the same system) and intersystem interference (interference from other systems) [2-4]. Field testing requires measurements using actual scenario, it cannot be conducted during the system development. Analytical techniques use an aggregate gain factor $\left(G_{a g g}\right)$ repeatedly in an interference analysis without resorting to the use of constellation simulation for every scenario. Although it can save the time consumption of computer, it seriously overstates the interference in most situations. Computer simulations can not only simulate every interference scenario for different user at every time and in every place but also provide more realistic results though it is more time-consuming.

WAAS is intended to enable aircraft to rely on GPS for all phases of flight including precision approaches to any airport within its coverage area. With using signal structures which are similar to other GNSS systems and sharing frequencies near to or overlapping those of GPS and WAAS radio frequency compatibility become a matter of great concern for the system providers and user community, this paper

*Corresponding author: $\underline{\mathrm{w} @ \text {,lliu.net }}$ mainly deals with the interference computation among the above-mentioned systems and displays some important radio frequency compatibility analysis results. In this paper, the computer simulations approach will be taken into account in the interference computation to pursue more realistic results. A more complete methodology is presented which accounts for the influence of the codes onto the spectra and the effects of Doppler frequency shift related to the Doppler velocity. Moreover, the power spectral density of the desired signal from the desired satellite will be considered, which was always neglected in the worse scenarios for the interference computation.

Interference will be mainly computed on the L1 band where GPS and Wide Area Augmentation System (WAAS) signals are sharing the same band. The maximum, minimum and mean intersystem interference results are important for the compatibility assessment of GNSS systems. However, the maximum result is enough in reality. In this paper, the worse scenarios are simulation using $5^{\circ} \times 5^{\circ}$ gird for longitude and latitude, and sampling the period time which is 60 seconds by small time steps.

This paper is organized as follows. In this paper, Section 2 describes a complete methodology which is used for interference simulation and computation. Section 3 introduces signal parameters and presents all the simulation and analysis results, while Sections 4 draws conclusions.

\section{METHODOLOGY}

\subsection{Interference assessment approach}

According to [1], a general quantity to reflect the effect of interference on characteristics at the input of the non-specific receiver was introduced. It was called the effective carrier power to noise density $\left(C / N_{0}\right)$, and denoted $\left(C / N_{0}\right)_{\text {eff }}$. When there are noise and one non-white interference signal, and the effect of filter- 
ing can be neglected within the pass-band, the effective $C / N_{0}$ can be written as follows:

$$
\left(\frac{C}{N_{0}}\right)_{e f f}=\frac{C \int_{-\beta_{r} / 2}^{\beta_{r} / 2} G_{s}(f) d f}{N_{0} \int_{-\beta_{r} / 2}^{\beta_{r} / 2} G_{s}(f) d f+C_{l} \int_{-\beta_{r} / 2}^{\beta_{r} / 2} G_{s}(f) G_{l}(f) d f}
$$

Where $G_{s}(f)$ is the normalized power spectral density of the desired signal; $C$ is the received power of the useful signal. $N_{0}$ is the power spectral density of the thermal noise. In this paper, we assume that $N_{0}$ is $-201.5 \mathrm{dBW} / \mathrm{Hz} ; G_{l}(f)$ is the normalized spectral density of the interference signal; $C_{l}$ is the received power of the interference signal; $\beta_{r}$ is the receiver front-end bandwidth.

The $\left(C / N_{0}\right)_{e f f}$ can be interpreted as the carrier power to noise density ratio caused by an equivalent white noise that would yield to the same correlation output variance obtained in presence of the interference signal. When there are the intrasystem interference and the intersystem interference, the $\left(C / N_{0}\right)_{\text {eff }}$ can be expressed as follows:

$$
\begin{aligned}
& \left(C / N_{0}\right)_{e f f}=\frac{C \int_{-\beta_{r} / 2}^{\beta_{r} / 2} G_{s}(f) d f}{N_{0} \int_{-\beta_{r} / 2}^{\beta_{r} / 2} G_{s}(f) d f+\sum_{i=1}^{M} \sum_{j=1}^{K_{i}} C_{i, j} \int_{-\beta_{r} / 2}^{\beta_{r} / 2} G_{s}(f) G_{i, j}(f) d f} \\
& =\frac{C}{N_{0}+\sum_{i=1}^{M} \sum_{j=1}^{K_{i}} C_{i, j} \frac{\int_{-\beta_{r} / 2}^{\beta_{r} / 2} G_{s}(f) G_{i, j}(f) d f}{\int_{-\beta_{r} / 2}^{\beta_{r} / 2} G_{s}(f) d f}} \\
& =\frac{C}{N_{0}+I_{G N S S}}
\end{aligned}
$$

Where:

$I_{G N S S}=\sum_{i=1}^{M} \sum_{j=1}^{K_{i}} C_{i, j} \frac{\int_{\beta_{r} / 2}^{\beta_{r} / 2} G_{s}(f) G_{i, j}(f) d f}{\int_{-\beta_{r} / 2}^{\beta_{r} / 2} G_{s}(f) d f}$

It denotes as follows:

$$
\lambda^{s}=\int_{-\beta_{r} / 2}^{\beta_{r} / 2} G_{s}(f) d f
$$

$I_{G N S S}$ is the aggregate equivalent noise power density of the combination of the intrasystem interference and the intersystem interference. $M$ is the visible number of satellites. $K_{i}$ is the number of signals transmitted by satellite $i . C_{i i}$ is the received power of the $j$-th interfering signal on the $i$-th satellite.

From (2), it is clear that the impact of the interference onto $\left(C / N_{0}\right)_{\text {eff }}$ is directly related to the spectral separation coefficient (SSC) of an interfering signal from the $j$-th interfering signal on the $i$-th satellite to desired signals. The SSC is defined as follows [2]:

$\kappa_{i, j}^{s}=\int_{-\beta_{r} / 2}^{\beta_{r} / 2} G_{s}(f) G_{i, j}(f) d f$

The spectral Separation Coefficient (SSC) parameter is appropriate to assess the impact of interfering signals on the prompt correlator channel of receiver at its processing phases (the acquisition, the carrier-phase tracking and the data demodulation) $[6,7]$. SSC is a parameter that indexes how the power spectral is overlapping; it is also a parameter to evaluate the intrasystem interference and the intersystem interference. What's more, it can evaluate the compatibility between different signals accurately.

When more than two systems are operated together, the aggregate equivalent noise power density $I_{G N S S}$ is the sum of two components:

$$
I_{\text {GNSS }}=I_{\text {Intra }}+I_{\text {Inter }}
$$

where $I_{\text {Intra }}$ is the equivalent noise power density of interfering signals from satellites belonging to the same system as the desired signal, and $I_{\text {Inter }}$ is the aggregate equivalent noise power density of interfering signals from satellites belonging to the other system.

A general method to calculate the $\left(C / N_{0}\right)_{e f f}$, an introduction of interfering signals from satellites belonging to the same system or other system is based on expression (2). In addition to the calculation of $\left(C / N_{0}\right)_{\text {eff }}$, the calculated degradation of effective $C / N_{0}$ is more interesting when more than two systems are operated together. The degradation of effective $C / N_{0}$ in the case of the inter-system interference is shown as follows [3]:

$$
\begin{aligned}
\Delta\left(C / N_{0}\right)_{\text {eff }}= & \frac{\frac{C}{N_{0}+I_{\text {Intra }}}}{\frac{C}{N_{0}+I_{\text {Intra }}+I_{\text {Inter }}}} \\
& =1+\frac{I_{\text {Inter }}}{N_{0}+I_{\text {Intra }}}
\end{aligned}
$$

Therefore, the expression of intrasystem interference in $\mathrm{dB}$ is shown as follows:

$\phi_{\text {Inter }}=10 \cdot \log \left(1+\frac{I_{\text {Inter }}}{N_{0}+I_{\text {Intra }}}\right)$

For the aggregate equivalent noise power density calculation, the constellation configuration, the satellite and user receiver antenna gain patterns, and the space loss are included in the link equation. The user receiver location must be taken into account for meas- 
uring the interference effects. When a receiver is in a given location $\mathrm{m}$ on the earth at any time over a time period, the aggregate equivalent noise power density to a desired signal can be written as follows:

$$
I_{m}^{s}(t)=\frac{1}{\lambda^{s}} \sum_{i=1}^{M(t)} \sum_{j=1}^{K_{i}} \frac{P_{i, j} G_{i}(t) G_{u s e r}(t)}{L_{d i s t}(t) L_{a t m} L_{p o l}} \kappa_{i, j}^{s}
$$

Where $P_{i j}$ is the transmit power of the $j$-th signal belonging to the $i$-th satellite; $G_{i}(t)$ is the satellite antenna gain between the $i$-th satellite and the user receiver at time $t ; G_{u s e r}(t)$ is the user receiver antenna gain between the user receiver and the $i$-th satellite at time t; $L_{\text {dist }}(t)$ is the loss of signal due to the distance $i$-th satellite and user at time $t ; L_{a t m}$ is the loss of the signal due to the atmospheric loss; $L_{p o l}$ is the polarization mismatch loss.

Note that Expression (9) is the sum of all equivalent noise power density from all signals of all satellites in view at any time. When the desired satellite is used, it must subtract the power spectral density of the desired signal from the desired satellite. Calculation of $I_{m}^{s}(t)$ for every interference scenario at every time in every place needs more time for simulations.

The atmospheric loss $L_{a t m}$ is estimated to be $0.5 \mathrm{~dB}$ for all signals and systems [3]. We assume that the WASS user receiver antenna is with $0 \mathrm{dBi}$ gain, but $3 \mathrm{dBi}$ gain for the GPS user receiver antenna. The polarization mismatch loss is assumed to be $4.0 \mathrm{~dB}$ and $3 \mathrm{~dB}$ respectively for GPS and WASS. The satellite antenna gain $G_{i}(t)$ is a function of the off-boresight angle $a$ [3], it can be illustrated in Figure 1:

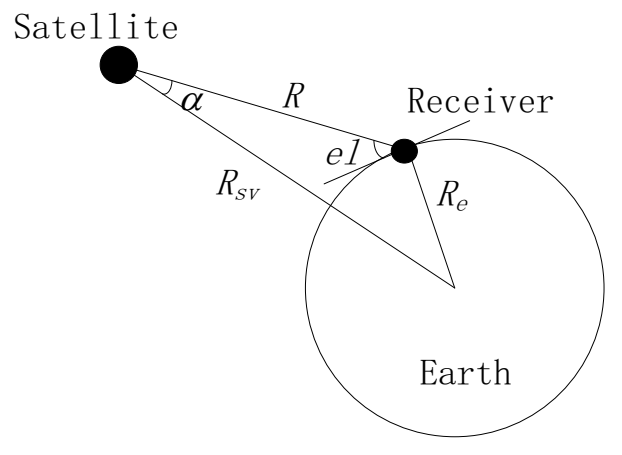

Figure 1. Illustration of off-boresight angle

The off-boresight angle $a$ can be calculated as a function of the satellite semi-major axis RSV, the Earth Radius Re and the satellite elevation angle at the user receiver el:

$$
\alpha=\sin ^{-1}\left[\frac{\mathrm{R}_{e} \sin \left(e l+90^{\circ}\right)}{R_{S V}}\right]
$$

Typical profile of GPS Block IIA satellite antenna gain is depicted in the Figure 2:

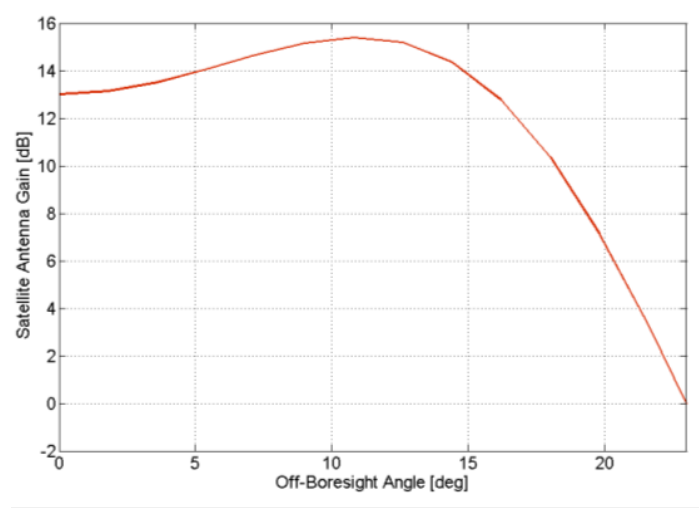

Figure 2. Typical satellite antenna gain

The signal distance loss $L_{\text {dist }}(t)$ can be expressed as follows:

$L_{\text {dist }}(t)=\left(\frac{c}{4 \pi d(t) f_{0}}\right)^{2}$

Where $c$ is the speed of light, $d(t)$ is the distance of satellite and user at time $t, f_{0}$ is the signal center frequency.

\subsection{Effects of the Doppler frequency shift}

The Doppler frequency shift caused by the satellite motion on the GNSS carrier frequency is important for the interference assessment. In this paper, the GNSS receiver is assumed to be stationary on the earth. The Doppler frequency shift is related to the Doppler velocity, it can be expressed as follows:

$f_{\text {Doppler }}=\frac{f_{0} v_{d}}{c}$

Where $v_{d}$ is the Doppler velocity, which is the component of the satellite velocity towards the user receiver.

When the Doppler frequency shift is considered, the $\left(C / N_{0}\right)_{\text {aff }}$ can be rewritten as follows:

$$
\left(C / N_{0}\right)_{e f f}=\frac{C}{N_{0}+\sum_{i=1}^{M} \sum_{j=1}^{K_{i}} C_{i, j} \frac{\int_{-\beta_{r} / 2}^{\beta_{r} / 2} G_{s}\left(f+f_{D_{s}}\right) G_{i, j}\left(f+f_{D_{i, j}}\right) d f}{\int_{-\beta_{r} / 2}^{\beta_{r} / 2} G_{s}\left(f+f_{D_{s}}\right) d f}}
$$

And the spectral separation coefficient (SSC) is shown as follows:

$\boldsymbol{\kappa}_{i, j}^{s}=\int_{-\beta_{r} / 2}^{\beta_{r} / 2} G_{s}\left(f+f_{D_{s}}\right) G_{i, j}\left(f+f_{D_{i, j}}\right) d f$ 
Where $f_{D}$ is the Doppler frequency shift of the desired signal; $f_{D_{i, j}}$ is the Doppler frequency shift of the $j$-th interfering signal on the $i$-th satellite.

\subsection{Influences of the codes}

In many cases, the line spectrum of short-code signal is often approximated by a continuous power spectral density (PSD) without fine structure. This approximation is valid for signals corresponding to long spreading code, but is not appropriate for short-code signals, for example, the $\mathrm{C} / \mathrm{A}$ code interference to other $\mathrm{C} / \mathrm{A}$ code signals [4]. For the case of GPS C/A-code with a data rate of $50 \mathrm{sps}$, the maximum value of real SPD is higher than the ideal smooth PSD. It is shown that the GPS C/A-code real SSC must be different from the analytical SSC. As we can see from Figure 3, GPS C/A-Code Self SSC is the function of Doppler frequency shift.

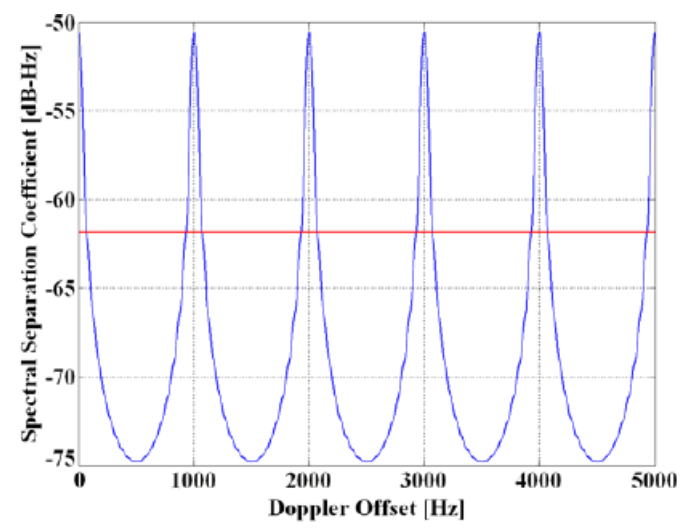

Figure 3. GPS C/A-Code Self SSC vs Doppler frequency shift

For different center frequency $f_{0}$, the Doppler frequency shift can be calculated by expression (12). Simulations have shown that the maximum Doppler frequency shift appears when the satellite is at the horizon position.

\section{SIMULATION AND RESULTS}

\subsection{Simulation parameters}

Table 1. Space constellations parameters

\begin{tabular}{lll}
\hline Parameter & GPS & WAAS \\
\hline & $24 / 6$ & There satellites: \\
Constellation & Plus 3 active & $98^{\circ} \mathrm{W} \quad 107.3^{\circ} \mathrm{W}$ \\
& spares. & $133^{\circ} \mathrm{W}$ \\
& & \\
Inclination (o) & 55 & 0 \\
Eccentricity & 0 & 0 \\
\hline
\end{tabular}

\begin{tabular}{l}
$\begin{array}{l}\text { Semi-major Ax- } \\
\text { is }(\mathrm{km})\end{array}$ \\
\hline
\end{tabular}

The space constellations parameters of GPS and WAAS are summarized in the Table 1.

For GPS, a 27-satellites constellation is taken in the interference simulation. The WAAS is an air navigation aid developed by the Federal Aviation Administration to augment the GPS, with the goal of improving its accuracy, integrity and availability. The WAAS space segment consists of three commercial satellites: Inmarsat-4 F3, Telesat's Anik F1R and Intelsat's Galaxy 15 [8].

It can be seen that a lot of attention must be paid to the signals spectrum overlaps at L1 band among these systems. Thus, we will only concentrate the interference on the L1 band in this study. All the L1 signals include: GPS C/A, L1C, P(Y) and M-code signals; the WAAS L1 C/A signal that will be taken into account in the simulations. All the L1 signals will be taken into account in the simulations. The following Table 2 is used to summarize the characteristics of GPS and WAAS signals to be transmitted in L1 band. The detailed information about the signal parameters can be respectively found in the GPS Interface Control Document [8].

Table 2. GPS and WAAS signal parameters in L1band

\begin{tabular}{lllll} 
System & $\begin{array}{l}\text { Service } \\
\text { Type }\end{array}$ & $\begin{array}{l}\text { Carrier } \\
\text { Frequency } \\
\text { (MHZ) }\end{array}$ & $\begin{array}{l}\text { Modulation } \\
\text { Type }\end{array}$ & $\begin{array}{r}\text { Chip Rate } \\
\text { (Mcps) }\end{array}$ \\
\hline \multirow{6}{*}{ GPS } & C/A & 1575.42 & BPSK & 1.023 \\
& P(Y) & 1575.42 & BPSK & 10.23 \\
& M & 1575.42 & BOC(10,5) & 5.115 \\
& L1C & 1575.42 & MBOC(6,1, & 1.023 \\
\multirow{2}{*}{ WASS } & L1 & 1575.42 & BPSK & 1.023 \\
\hline
\end{tabular}

3.2 Simulation results

Table 3. Simulation parameters

\begin{tabular}{ll}
\hline Parameter & Value \\
\hline Time period & 10 days \\
Time step & 60 second \\
Grid Resolution & $5^{\circ} \times 5^{\circ}$ \\
Elevation Angle & $5^{\circ}$ \\
& GPS: $30.69 \mathrm{MHz}$ \\
Emission Bandwidth & WAAS: $30.69 \mathrm{MHz}$ \\
Front End Bandwidth & $40.92 \mathrm{MHz}$ \\
\hline All interference simulation results are referred to
\end{tabular}
the worse case scenarios. The worse scenarios are assumed as the minimum emission power for desired signal, the maximum emission power for all interfered signals and the maximum $\left(C / N_{0}\right)_{\text {eff }}$ degradation of interference over all time steps. It must be noted that the mean case scenarios and better scenarios are also 
used in the interference calculation. However, in this paper, we only show the results for the worse case scenarios.

The following table summarizes the simulation parameters that have been considered in this paper.

Figure 4 and Figure 5 show the maximum $C / N_{0}$ degradation of GPS civil signals due to the WAAS intersystem interference in L1 band. It can be seen that the intersystem degradations on L1C signal is very small and never greater than or equal to $0.08 \mathrm{~dB}$. The GPS C/A-code signal degradation is raised from $0 \mathrm{~dB}$ to $0.25 \mathrm{~dB}$. Thus, we can see the WAAS signal has a very small effect on the GPS L1C civil signal.

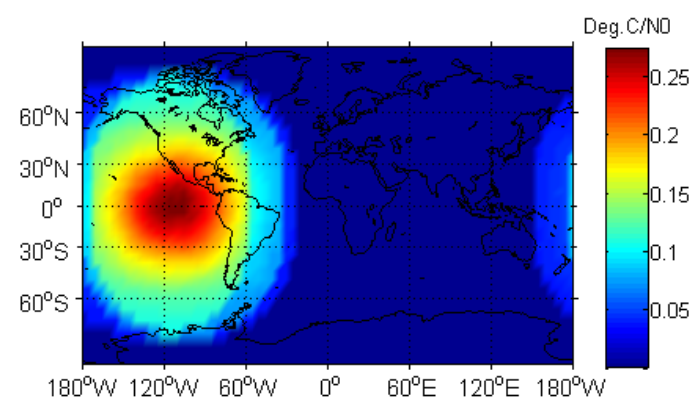

Figure 4. Max. $C / N_{0}$ degradation of GPS C/A-code due to WAAS intersystem interference in $\mathrm{L} 1$ band

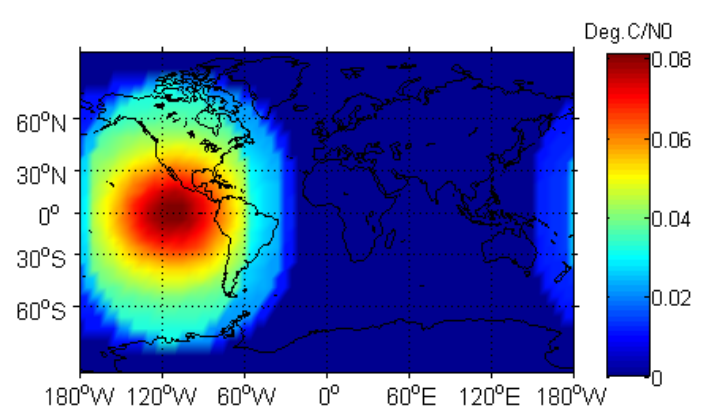

Figure 5. Max. $C / N_{0}$ degradation of GPS L1C signal due to WAAS intersystem interference in $\mathrm{L} 1$ band

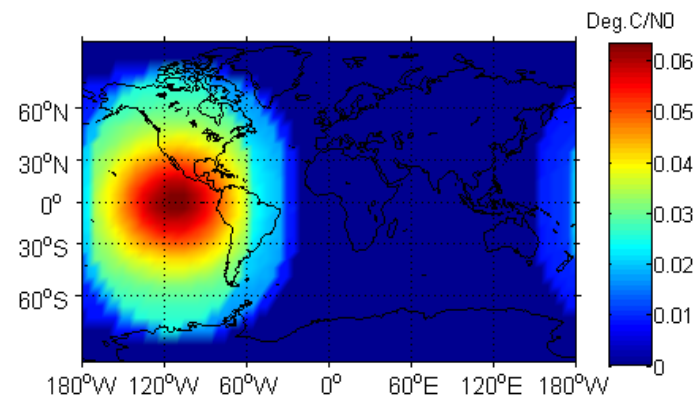

Figure 6. Max. $C / N_{0}$ degradation of GPS L1P signal due to WAAS intersystem interference in $\mathrm{L} 1$ band

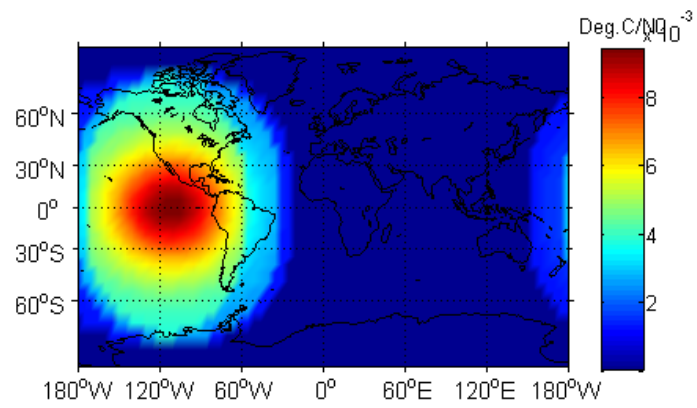

Figure 7. Max. $C / N_{0}$ degradation of GPS L1 M-code signal due to WAAS intersystem interference in L1 band

In the cases that GPS military signals interfered by WAAS are presented in Figure 6 and Figure 7. The maximum value $C / N_{0}$ degradation of GPS L1P signal is increased to $0.06 \mathrm{~dB}$, and the intersystem degradation on L1 M-code is very small.

The maximum $C / N_{0}$ degradation result of WAAS signal due to GPS signals in L1 band is also shown in Figure 8. The maximum value of $C / N_{0}$ degradation of WAAS L1 signal is increased to $2.82 \mathrm{~dB}$. A significant increase of the maximum value due to more signals in GPS L1 band, especially M-code and P-code emission power, are high in the worse scenario.

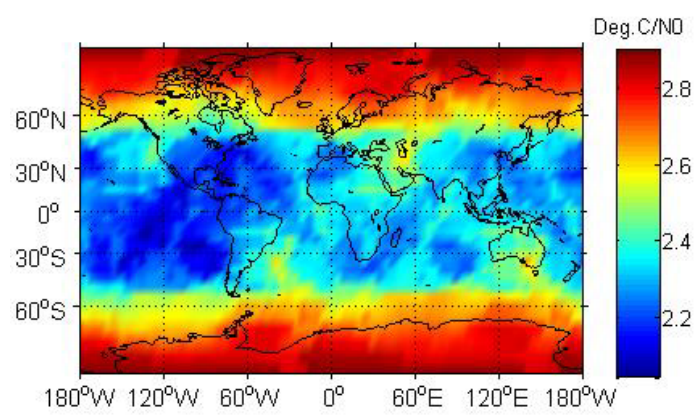

Figure 8. Max. $C / N_{0}$ degradation of WASS L1 signal due to GPS intersystem interference on L1 band

All the results are shown that the introduction of the WAAS system leads to the intersystem interference for the GPS. But the values are smaller than the GPS interfered with WAAS. It can be found that the WAAS provides a sound foundation for compatibility with GPS. 


\section{CONCLUSIONS}

In this paper, a complete methodology for the radio frequency compatibility assessment has been described. A detailed derivation of the methodology including equations and computation principle was provided. Real simulations accounting for the interference effects were carried out every time and in every place on the earth for L1 band where GPS and WAAS share the same band. It was shown that the introduction of the WASS system leads to intersystem interference for the GPS. Though the introduction of the WASS system leads to the intersystem interference degradation of $C / N_{0}$ increasing, the value is very small. As a conclusion, the WASS provides a sound basis for compatibility with GPS. Advantages of multi-mode GNSS receivers would benefit the user community. At the end, it has to be pointed that interference results shown in this work are mainly referring to worst case calculations. Though the value is higher than normal value, it is feasible for the interference assessment of GNSS system.

\section{ACKNOWLEDGEMENTS}

This paper is sponsored by Science and Technology Program of Shanghai Maritime University (GN: 20120092).

\section{REFERENCES}

[1] Betz, J. \& M.C.B. MA. 2001. Effect of partial-band interference on receiver estimation of C/NO: Theory. Defense Technical Information Center.

[2] Betz, J.W. 1999. The Offset Carrier Modulation for GPS Modernization, Proc. ION 1999 National Technical Meeting.

[3] Wallner, S., et al. 2005. Interference Computations Between GPS and GALILEO. Proceedings of the 18th International Technical Meeting of the Satellite Division of the Institute of Navigation.

[4] Van Dierendonck, A. \& Hegarty, C. 2002. Methodologies for Assessing Intrasystem and Intersystem Interference to Satellite Navigation Systems. Institute of Navigation, 3975 University Drive, Suite 390, Fairfax, VA, 22030, USA.

[5] Titus, B., Betz, J. W., Hegarty, C. J. \& Owen, R. 2003. Intersystem and intrasystem interference analysis methodology. Proc. ION GNSS 2003, September 9 - 12, 2003, Portland, OR, 2061-2069.

[6] Godet J., de Mateo J. C., Erhard P. \& Nouvel O. 2002. Assessing the Radio Frequency Compatibility between GPS and Galileo. ION GPS 2002, 24-27 September 2002, Portland, OR.

[7] Owen, R., Goldstein, D. B. \& Hegarty, C.J. 2002. Modeling Maximum Aggregate GPS Signal Power Levels for GPS Self-Interference Analyses. Proc. ION NTM 2002, January 28 - 30, 2002, San Diego, CA, 939 - 947.
[8] Navstar GPS Space Segment/Navigation User Interfaces. 2006. 